Fixed Point Theory, 20(2019), No. 2, 483-506

DOI: $10.24193 /$ fpt-ro.2019.2.31

http://www.math.ubbcluj.ro/ ${ }_{\text {nodeacj/sfptcj.html }}$

\title{
NIELSEN THEORY ON INFRA-NILMANIFOLDS MODELED ON THE GROUP OF UNI-TRIANGULAR MATRICES
}

\author{
YOUNGGI CHOI*,1, JONG BUM LEE**,2 AND KYUNG BAI LEE*** \\ *Department of Mathematics Education, Seoul National University \\ Seoul 08826, Korea \\ E-mail: yochoi@snu.ac.kr \\ ** Department of Mathematics, Sogang University \\ Seoul 04107, Korea \\ E-mail: jlee@sogang.ac.kr \\ *** Department of Mathematics, University of Oklahoma \\ Norman, OK 73019, USA \\ E-mail: kblee@math.ou.edu
}

\begin{abstract}
Let $\mathrm{Nil}_{m}$ be the group of $m \times m$ uni-triangular matrices. Then it is a connected and simply connected $(m-1)$-step nilpotent Lie group. Using the averaging formulas, we compute the spectra of the Lefschetz, Nielsen and Reidemeister (coincidence) numbers of maps on infra-nilmanifolds modeled on $\mathrm{Nil}_{m}$. As a byproduct, we prove that the Bieberbach groups of $\mathrm{Nil}_{m}(m \geq 4)$ with $\Gamma_{m}$ as its nil-radical satisfy the $R_{\infty}$ property.

Key Words and Phrases: Averaging formula, infra-nilmanifold, Lefschetz number, Nielsen number, Reidemeister number, uni-triangular matrix.
\end{abstract}

2010 Mathematics Subject Classification: 57S30, 57S20, 22E25.

\section{REFERENCES}

[1] R.F. Brown, On a homotopy converse to the Lefschetz fixed point theorem, Pacific J. Math., 17(1966), 407-411.

[2] Y. Cao, Z. Tan, Automorphisms of the Lie algebra of strictly upper triangular matrices over a commutative ring, Linear Algebra Appl., 360(2003), 105-122.

[3] Y. Choi, J.B. Lee, K.B. Lee, Infra-nilmanifolds modeled on the group of uni-triangular matrices, Geom. Dedicata, 189(2017), 161-176.

[4] K. Dekimpe, P. Igodt, S. Kim, K.B. Lee, Affine structures for closed 3-dimensional manifolds with Nil-geometry, Quart. J. Math. Oxford Ser. (2), 46(1995), 141-167.

[5] A. Fel'shtyn, J.B. Lee, The Nielsen and Reidemeister numbers of maps on infra-solvmanifolds of type (R), Topology Appl., 181(2015), 62-103.

\footnotetext{
${ }^{1}$ The first-named author was supported by Basic Science Research Program through the National Research Foundation of Korea(NRF) (NRF-2013R1A1A2006926).

${ }^{2}$ The second-named author was supported by Basic Science Researcher Program through the National Research Foundation of Korea(NRF) (NRF-2016R1D1A1B01006971).
} 
[6] K.Y. Ha, J.B. Lee, P. Pennincks, Formulas for the Reidemeister, Lefschetz and Nielsen coincidence number of maps between infra-nilmanifolds, Fixed Point Theory Appl., 39(2012), $1-23$.

[7] S.W. Kim, J.B. Lee, Averaging formula for Nielsen coincidence numbers, Nagoya Math. J., 186(2007), 69-93.

[8] S.W. Kim, J.B. Lee, K.B. Lee, Averaging formula for Nielsen numbers, Nagoya Math. J., 178(2005), 37-53.

[9] K.B. Lee, Maps of infra-nilmanifolds, Pacific J. Math., 168(1995), no. 1, 157-166.

[10] J.B. Lee, K.B. Lee, Lefschetz numbers for continuous maps, and periods for expanding maps on infra-nilmanifolds, J. Geom. Phys., 56(2006), 2011-2023.

[11] M. Newman, Integral Matrices, Pure and Applied Mathematics, Vol. 45, Academic Press, New York-London, 1972.

Received: December 21, 2016; Accepted: May 18, $201 \%$. 